\title{
circRNA_001275 upregulates Wnt7a expression by competitively sponging miR-370-3p to promote cisplatin resistance in esophageal cancer
}

\author{
FANG-WEN ZOU ${ }^{1}$, SHI-ZE YANG ${ }^{2}$, WEN-YA LI ${ }^{2}$, CHAO-YUAN LIU ${ }^{1}$, \\ XU-HONG LIU ${ }^{1}$, CHUN-HONG HU ${ }^{1}$, ZHENG-HUA LIU ${ }^{2}$ and SHUN XU ${ }^{2}$ \\ ${ }^{1}$ Department of Oncology, The Second Xiangya Hospital of Central South University, Changsha, Hunan 410000; \\ ${ }^{2}$ Department of Thoracic Surgery, The First Affiliated Hospital of China Medical University, \\ Shenyang, Liaoning 11000, P.R. China
}

Received July 20, 2019; Accepted February 5, 2020

DOI: $10.3892 /$ ijo.2020.5050

\begin{abstract}
Circular RNAs (circRNAs) are aberrantly expressed in various tumors and are associated with tumorigenesis. The present study aimed to determine the role of circRNA_001275 in cisplatin-resistant esophageal cancer. Three pairs of cisplatin-resistant tissues and corresponding adjacent tissues were collected and subjected to circRNA chip analysis. Additionally, the effect of circRNA_001275 on cisplatin-resistant cells was investigated. The relationship between circRNA_001275, microRNAs (miRs) and target genes were analyzed using luciferase assays, and validated via reverse transcription-quantitative PCR (RT-qPCR) and western blotting. The results showed that circRNA_001275 was significantly upregulated in cisplatin-resistant esophageal cancer tissues and cells $(\mathrm{P}<0.05)$. Overexpression of circRNA_001275 promoted the proliferation and invasion, and decreased the apoptosis of cisplatin-resistant cells. On the other hand, circRNA_001275 silencing inhibited cell proliferation and invasion, and promoted cell apoptosis $(\mathrm{P}<0.05)$. Dual-luciferase reporter assays revealed that circRNA_001275 directly binds to miR-370-3p, and that Wnt family member 7A (Wnt7a) is targeted by miR-370-3p. RT-qPCR and western blotting further demonstrated that circRNA_001275 serves as an miR-370-3p sponge to upregulate Wnt7a expression. In conclusion, the present study revealed that circRNA_001275 was upregulated in cisplatin-resistant esophageal cancer and promoted cisplatin
\end{abstract}

Correspondence to: Dr Zheng-Hua Liu, Department of Thoracic Surgery, The First Affiliated Hospital of China Medical University, 155 North Nanjing Street, Heping, Shenyang, Liaoning 11000, P.R. China

E-mail: zhliu@cmu.edu.cn

Key words: esophageal cancer, cisplatin resistance, circular RNA_001275, microRNA-370-3p, Wnt family member 7A resistance by sponging miR-370-3p to upregulate Wnt7a expression. Therefore, circRNA_001275 may serve as a potential diagnostic biomarker and therapeutic target for patients with cisplatin-resistant esophageal cancer.

\section{Introduction}

At present, $\sim 1 / 3$ of esophageal cancer cases are diagnosed at an advanced stage, and are no longer eligible for surgical resection (1). Chemotherapy is the main treatment for advanced esophageal cancer, and cisplatin-based chemotherapy is considered to be a first-line therapy (2). However, the majority of patients will benefit from cisplatin treatment for the initial 4-6 chemotherapy cycles, then subsequently develop cisplatin resistance $(3,4)$. Cisplatin resistance is the main reason for treatment failure and mortality in patients with esophageal cancer (5).

Circular RNAs (circRNAs) are a novel class of endogenous non-coding RNAs characterized by a covalently closed loop without 5' caps and 3' tails (6). circRNAs are conserved and stable, and may therefore serve as suitable markers for disease diagnosis and treatment; previous studies have shown that circRNAs serve important roles in various tumors, and the abnormal expression of circRNAs may be involved in the occurrence and development of cancer $(7,8)$. Furthermore, circRNAs may act as microRNA (miRNA/miR) sponges by competitively binding to miRNA response elements to influence downstream target gene expression, as well as affecting gene function at a post-translational level (9).

The present study aimed to investigate the role of circRNAs in cisplatin-resistant esophageal cancer. A circRNA chip assay revealed that circRNA_001275 was significantly upregulated in cisplatin-resistant esophageal cancer. Furthermore, circRNA_001275 was found to serve as an miR-370-3p sponge, upregulating Wnt family member 7A (Wnt7a) expression and consequently promoting cisplatin resistance. To the best of our knowledge, the present study was the first to demonstrate the role of the circRNA_001275/miR-370-3p/Wnt7a axis in cisplatin-resistant esophageal cancer. 


\section{Materials and methods}

Cell lines. The parental cisplatin-sensitive cells lines KYSE30 and ECA109 and the corresponding cisplatin-resistant cell lines KYSE30/DDP and ECA109/DDP were purchased from The Cell Bank of Type Culture Collection of the Chinese Academy of Sciences. The cells after resuscitation were cultured in high-glucose DMEM containing 10\% FBS (both Sigma-Aldrich; Merck $\mathrm{KGaA}$ ) and incubated at $37^{\circ} \mathrm{C}$ with $5 \% \mathrm{CO}_{2}$.

Clinical specimens. Three pairs of pathologically confirmed cisplatin-resistant and adjacent cisplatin-sensitive tissue were collected from patients with esophageal cancer at the Department of Thoracic Surgery of The First Affiliated Hospital of China Medical University between May 2017 to March 2018. The clinicopathological characteristics of the three patients are presented in Table SI. The present study was approved by the Ethics Committee of The First Affiliated Hospital of China Medical University and performed in accordance with the Declaration of Helsinki (10). Informed consent was obtained from all patients.

circRNA chip detection. The circRNA expression profiles of the three pairs of cisplatin-resistant and corresponding adjacent tissues were evaluated using a circRNA chip assay (ArrayStar Co., Ltd.). The assay was performed and analyzed by Shanghai GeneChem Co., Ltd.

Reverse transcription-quantitative PCR (RT-qPCR). Total RNA was extracted using TRIzol ${ }^{\circledR}$ reagent (Invitrogen; Thermo Fisher Scientific, Inc.) and reversed transcribed into cDNA using a riboSCRIPT ${ }^{\mathrm{TM}}$ reverse transcription kit (Guangzhou RiboBio Co., Ltd.). RT was conducted at $42^{\circ} \mathrm{C}$ for $60 \mathrm{~min}$ and $70^{\circ} \mathrm{C}$ for $10 \mathrm{~min}$. The qPCR primers were designed using Primer Premier software (version 5; Premier Biosoft International) and synthesized by Shanghai GeneChem Co., Ltd. The following primers were used: circRNA_001275 forward, 5'-TCTTCTTCTCCACTCCTGAA-3' and reverse, 5'-GAGCAAGGGCCCTAGCTCAA-3'; miR-370-3p forward, 5'-TGTAACCAGAGAGCGGGATGT-3' and reverse, 5'-TTT TGGCATAACTAAGGCCGAA-3'; Wnt7a forward, 5'-CTC CGGATCGGTGGCTATG-3' and reverse, 5'-CCCATTTGT GAGCCTTCTCCT-3'; U6 forward, 5'-CTCGCTTCGGCA GCACA-3' and reverse 5'-AACGCTTCACGAATTTGC GT-3'; and GAPDH forward, 5'-CGTCACCAACTGGGACGA CA-3' and reverse, 5'-CTTCTCGCGGTTGGCCTTGG-3'. The qPCR thermocycling conditions were as follows: $94^{\circ} \mathrm{C}$ for $15 \mathrm{sec}$, then $55^{\circ} \mathrm{C}$ for $30 \mathrm{sec}$ and $70^{\circ} \mathrm{C}$ for $30 \mathrm{sec}$ for 40 cycles. mRNA levels were quantified using the $2^{-\Delta \Delta C q}$ method (11); circRNA_001275 and Wnt7a were normalized to GAPDH, whereas miR-370-3p was normalized to U6.

Cell transfection. The circRNA_001275 overexpression (OE) vector (circRNA_001275 OE), pcDNA3.1 empty vector, small interfering (si)RNA targeting circRNA_001275 (si-circRNA_001275), si-negative control (NC), miR-370-3p mimic, miR-370-3p inhibitor and miR-370-3p NC labeled with green fluorescent protein were designed and synthesized by Shanghai GeneChem Co., Ltd. Sequences were as follows: si-circRNA_001275, 5'-GTTGAAGGGGGA
GCTCCTGTCATAAAAGCCAA-3'; si-NC, 5'-GAGCCC CAGCCTTCTCCATG-3'. miR-370-3p mimic, 5'-GCC TGCTGGGGTGGAACCTGGT-3'; miR-370-3p inhibitor, 5'-CAGGUCACGUCUCUGCAGUUAC-3'; and miR-370-3p NC, 5-GCC TGCTGGGGTGGA ACCTCCT-3'. Cells (1x10 1 well) were transfected with $50 \mathrm{nM} \mathrm{RNA}$ or plasmid using Lipofectamine ${ }^{\circledR} 2000$ (Invitrogen; Thermo Fisher Scientific, Inc.). At $48 \mathrm{~h}$ later, the transfection efficiency was assessed by RT-qPCR. The circRNA_001275 OE vector or pcDNA3.1 empty vector were transfected into KYSE30/DDP cells, whereas si-circRNA_001275 or si-NC were transfected into ECA109/DDP cells.

MTT assay. Transfected cells in the logarithmic growth phase were digested with trypsin at room temperature for $5 \mathrm{sec}$ and seeded into 96 -well plates at a density of $1 \times 10^{4}$ cells/well. A total of $20 \mu \mathrm{l}$ MTT solution $(2.5 \mathrm{mg} / \mathrm{ml})$ was added to the cells at $0,12,24,48$ and $72 \mathrm{~h}$. The cells were subsequently incubated for an additional $4 \mathrm{~h}$ at $37^{\circ} \mathrm{C}$ with $5 \% \mathrm{CO}_{2}$. The purple formazan crystals were dissolved by the addition of $100 \mu \mathrm{l} /$ well DMSO (Guangzhou RiboBio Co., Ltd.) and the absorbance was measured at a wavelength of $490 \mathrm{~nm}$.

Hoechst 33258 staining. Transfected cells in the logarithmic growth phase were digested with trypsin and seeded into 6-well plates at a density of $5 \times 10^{5}$ cells/well. Following incubation for $24 \mathrm{~h}$ at $37^{\circ} \mathrm{C}$, the cells were fixed with $3 \%$ paraformaldehyde for $5 \mathrm{~min}$ at $37^{\circ} \mathrm{C}$ and washed twice with PBS. The cells were stained with $10 \mu$ l Hoechst 33258 for 15 min in the dark at $37^{\circ} \mathrm{C}$ and subsequently sealed with neutral balsam. Apoptotic cells in five randomly selected fields imaged under a fluorescence microscope (magnification, $\mathrm{x} 400$ ).

Luciferase assay. The 3'-untranslated region (3'-UTR) of circRNA_001275 and Wnt7a were cloned into the XbaI site of the pGL3 control vector (Promega Corporation). Cells were co-transfected with the miR-370-3p mimic or miR-370-3p NC along with wild-type (wt) or mutated (mut) circRNA_001275-3'-UTR or Wnt7a-3'-UTR using Lipofectamine 2000 at a final concentration of $50 \mathrm{nM}$ for each RNA/plasmid. Firefly and Renilla luciferase activity was detected using a dual-luciferase reporter assay system (Promega Corporation). Firefly luciferase activity was normalized to Renilla luciferase activity (Promega Corporation). The luciferase assay was performed by Shanghai GeneChem Co., Ltd.

Prediction of target genes. Target genes were predicted using miRNA target gene prediction databases. miRanda v5 (http://www.microrna.org/microrna/home.do), TargetScan v7.1 (http://www.targetscan.org) and miRBase (http://www. mirbase.org/). Genes which appeared in $>2$ databases simultaneously were predicted to be target genes.

Western blotting. Transfected cells in the logarithmic growth phase were digested, and total protein was collected using RIPA buffer (Beijing Solarbio Science \&Technology Co., Ltd.) with $1 \mathrm{mM}$ phenylmethylsulfonyl fluoride and subsequently quantified using a bicinchoninic acid protein assay kit. Total protein (50 $\mu \mathrm{g} /$ lane) was separated via 10\% SDS-PAGE and then transferred onto PVDF membranes. The membranes were 

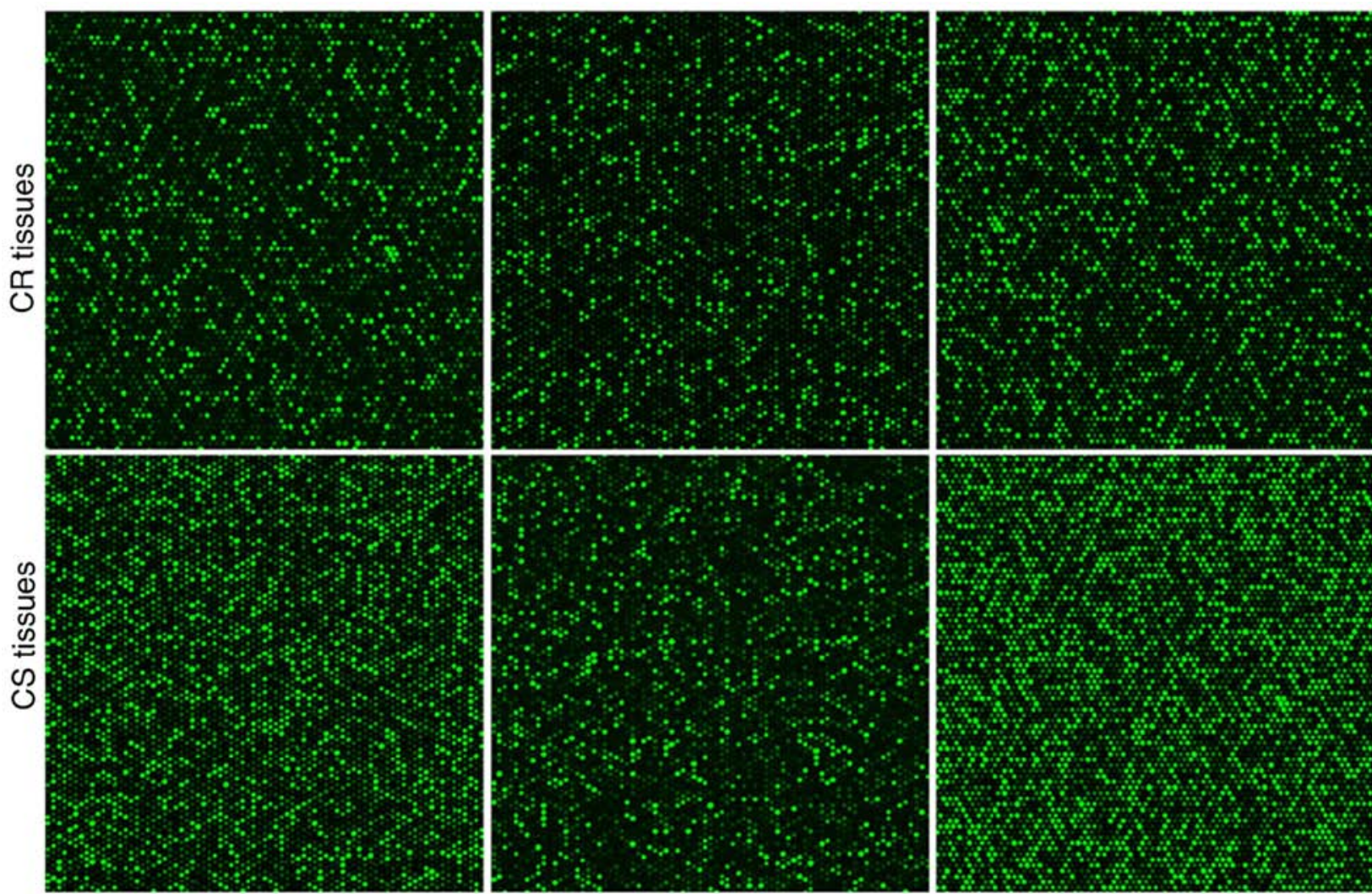

Figure 1. circRNA expression profiles of CR tissues as evaluated by a circRNA chip assay. circRNA microarray hybridization signal diagram. circRNA, circular RNA; CS, cisplatin-sensitive; CR, cisplatin-resistant.

blocked for $1 \mathrm{~h}$ in blocking buffer with $5 \%$ non-fat milk at room temperature. Subsequently, the PVDF membranes were incubated with primary antibodies against Wnt7a (1:1,000; cat. no. sc-365665; Santa Cruz Biotechnology, Inc.) and b-actin (1:1,000; cat. no. sc-69879; Santa Cruz Biotechnology, Inc.) overnight at $4^{\circ} \mathrm{C}$. Following primary antibody incubation, the membranes were incubated with horseradish peroxidase-conjugated secondary antibody (1:1,500; cat. no. sc-516102; Santa Cruz Biotechnology, Inc.). The membranes were washed twice with PBS and protein bands were visualized using a ECL-PLUS kit (GE Healthcare Life Sciences). Densitometry analysis was performed using Image J software v1.5 (National Institutes of Health) with $\beta$-actin as the loading control.

Statistical analysis. Statistical analyses were performed using SPSS software (version 17; SPSS, Inc.). Data are presented as the mean \pm SD of three experimental repeats. Student's t-test was used to analyze the differences between two groups. One-way ANOVA followed by Tukey's multiple comparison test was used to analyze differences among three groups. $\mathrm{P}<0.05$ was considered to indicate a statistically significant difference.

\section{Results}

circRNA expression profiles. circRNA expression profiles were evaluated using a circRNA chip assay. Polyacrylamide gel electrophoresis revealed that the total RNA obtained from the three pairs of tissues was of high purity and undegraded (Fig. 1A). The circRNA microarray hybridization signal diagram of the three tissue pairs acquired by the circRNA chip scanner is presented in Fig. 1B. The box plot (Fig. 1C), volcano plot (Fig. 2A) and scatter plot (Fig. 2B) revealed variable circRNA expression.

circRNA_001275 is significantly upregulated in cisplatinresistant esophageal cancer. The cluster heat map (Fig. 3A) revealed the top ten most upregulated and downregulated circRNAs. circRNA_001275 was the most highly upregulated (14.85-fold) circRNA in cisplatin-resistant tissues with highest raw intensity. Therefore, circRNA_001275 was selected as the primary focus of the study. The circRNA chip results were validated via RT-qPCR; circRNA_001275 was upregulated in cisplatin-resistant tissues (Fig. 3B), as well as KYSE30/DDP and ECA109/DDP cells ( $\mathrm{P}<0.05$; Fig. 3C), compared with corresponding adjacent tissues and sensitive cells.

Transfection efficiency. Fluorescence micrographs were captured at 24 and 48 h post-transfection. KYSE30/DDP and ECA109/DDP cells appeared green under a fluorescence microscope (Fig. 4A). RT-qPCR validation demonstrated that circRNA_141539 expression was significantly increased in the circRNA_001275 OE group, and significantly reduced in the si-circRNA_001275 group, compared with the corresponding control groups (both $\mathrm{P}<0.05$; Fig. 4B).

circRNA_001275 promotes cell proliferation. MTT and EdU assays were used to detect the effects of circRNA_001275 on cell proliferation. The results showed that cell viability $(\mathrm{P}<0.05$; Fig. 5A) and the ratio of EdU-positive cells $(\mathrm{P}<0.05$; Fig. $5 \mathrm{~B})$ 

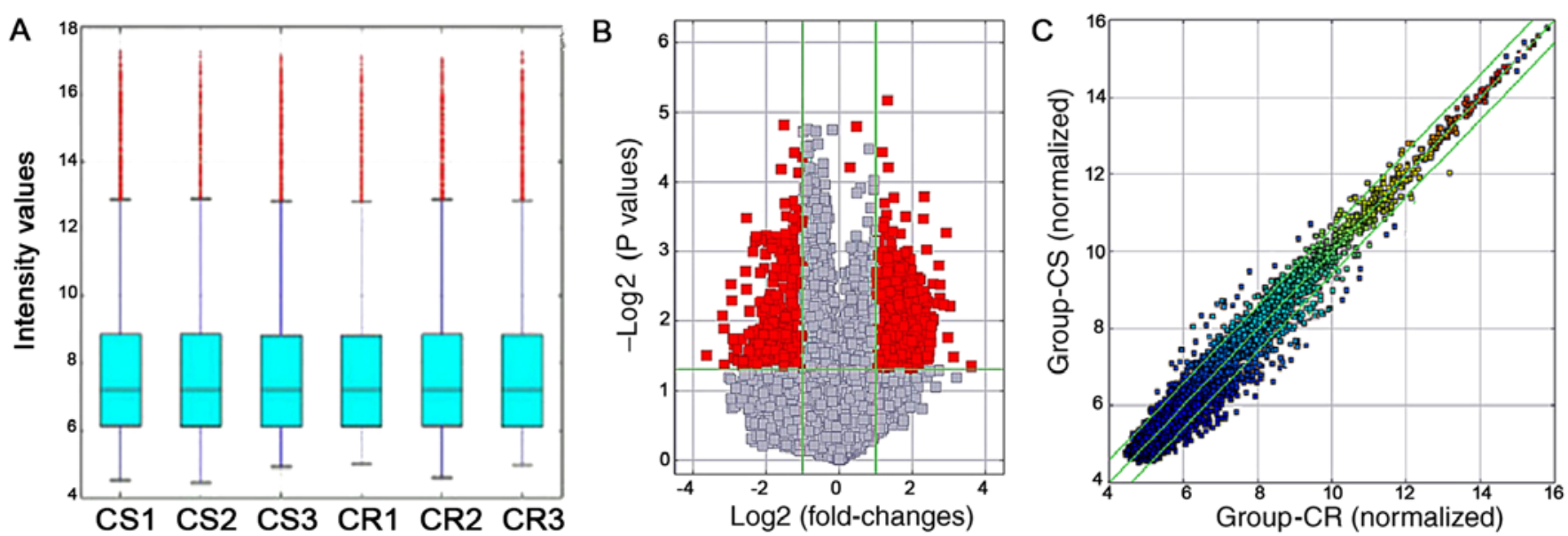

Figure 2. Differential expression of circRNAs in CR and CS tissues. (A) Box plot, (B) volcano plot and (C) scatter plot of circRNAs. circRNA, circular RNA; CS, cisplatin-sensitive; CR, cisplatin-resistant.
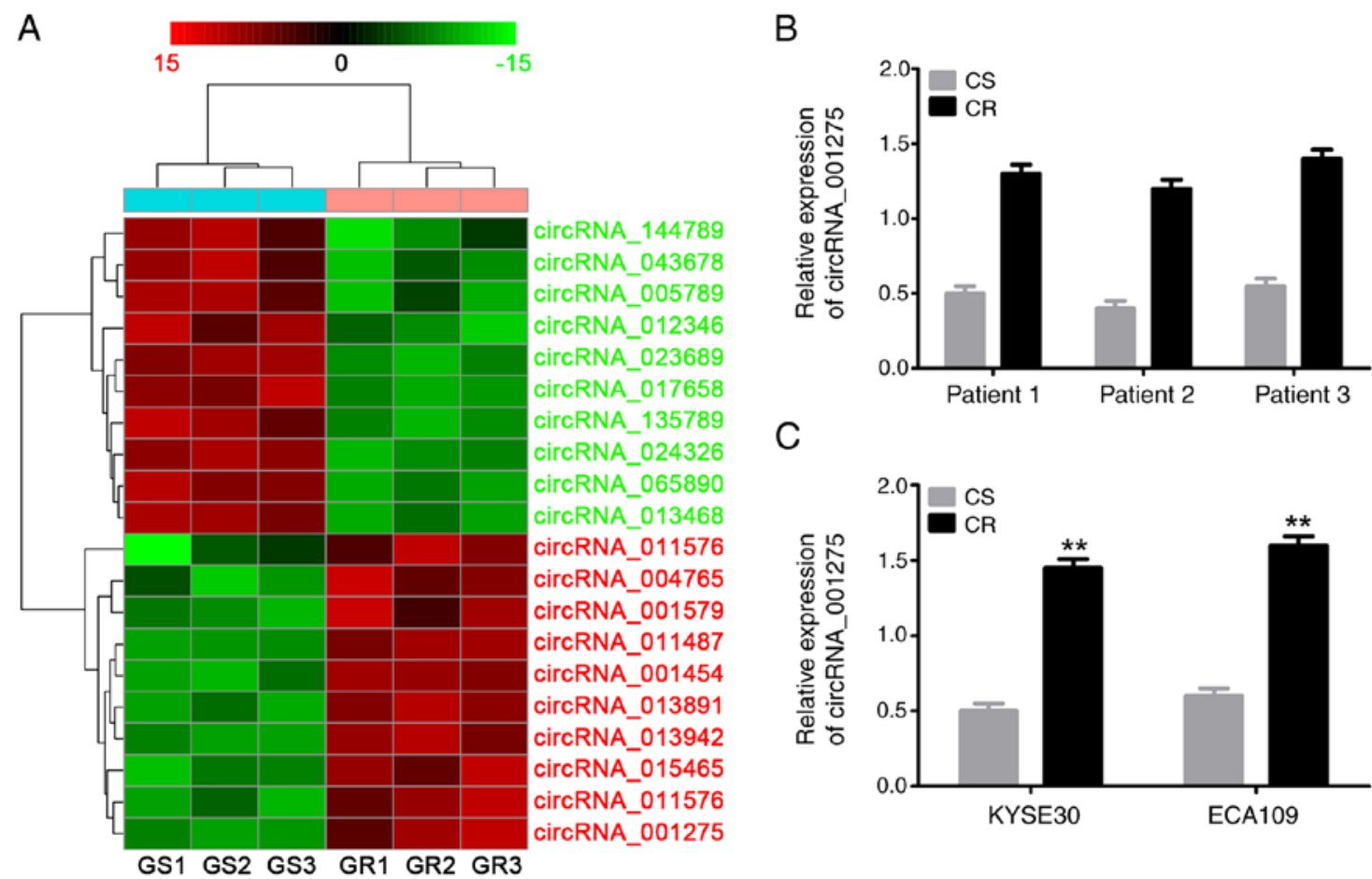

C

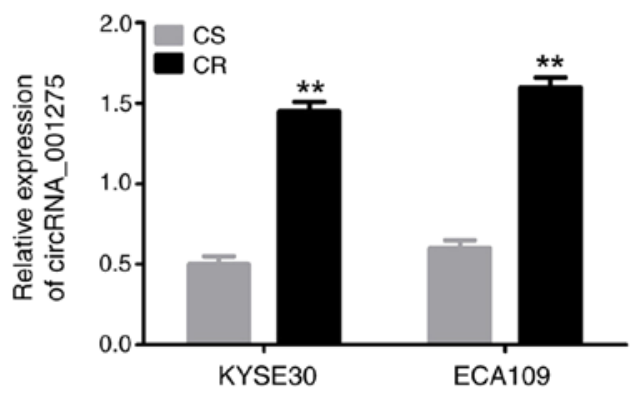

Figure 3. circRNA_001275 is upregulated in esophageal cancer with cisplatin resistance. (A) Cluster heat map of differentially expressed circRNAs. (B) RT-qPCR analysis circRNA_001275 expression in tissues. (C) RT-qPCR analysis of circRNA_001275 expression in cell lines. circRNA_001275 expression levels were normalized to GAPDH. N=3. ${ }^{* *} \mathrm{P}<0.01$ vs. CS. circRNA, circular RNA; CS, cisplatin-sensitive; CR, cisplatin-resistant; RT-qPCR, reverse transcription-quantitative PCR.

were significantly increased in the circRNA_001275 OE group, and significantly decreased in the si-circRNA_001275 group (both $\mathrm{P}<0.05$ ), compared with the corresponding control groups.

circRNA_001275 promotes cell invasion and inhibits cell apoptosis. Transwell invasion assays and Hoechst 33258 staining were used to detect the effects of circRNA_001275 on cell invasion and apoptosis, respectively. The apoptosis rate was significantly decreased in the circRNA_001275 OE group, and significantly increased in the si-circRNA_001275 group, compared with the corresponding control group (both $\mathrm{P}<0.05$; Fig. 6).

circRNA_001275 serves as an miR-370-3p sponge. A schematic representation of the potential binding sites between miR-370-3p and the circRNA_001275-3'-UTR is presented in Fig. 7A. miR-370-3p NC, mimic or inhibitor were transfected into KYSE30/DDP and ECA109/DDP cells. RT-qPCR results confirmed that miR-370-3p were significantly increased in the miR-370-3p mimic group, and significantly reduced in miR-370-3p inhibitor group, compared with the 
A

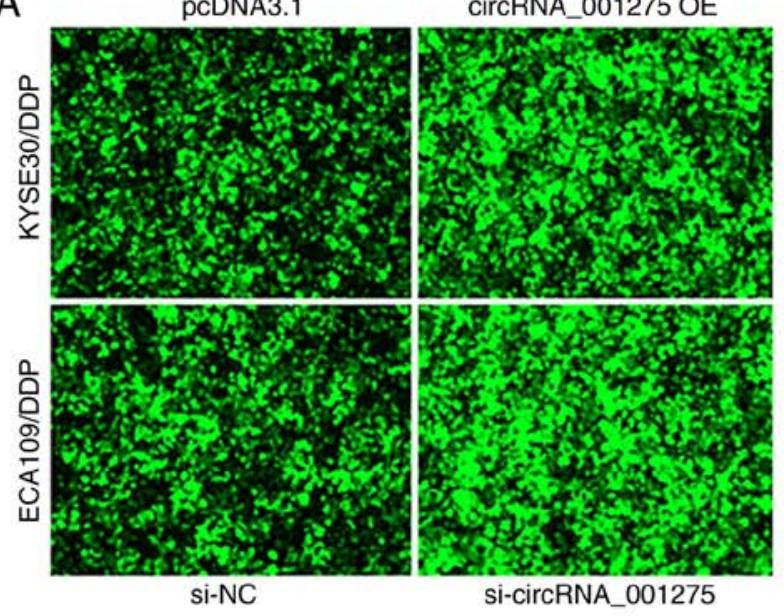

B

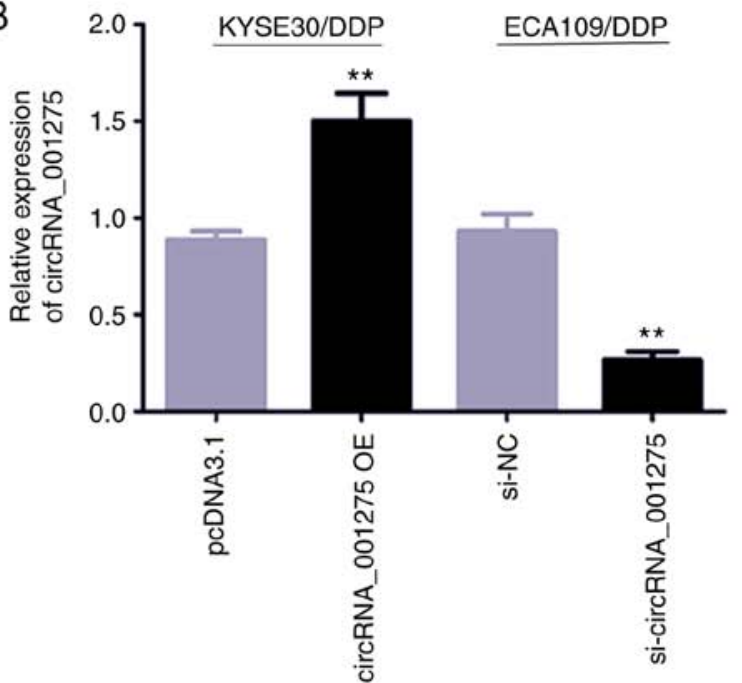

Figure 4. Transfection efficiency of circRNA 001275 OE and siRNA vectors. (A) Green color of cells under a fluorescence microscope. (B) Reverse transcription-quantitative PCR analysis of circRNA_001275 expression in each group. $\mathrm{N}=3 .{ }^{* *} \mathrm{P}<0.01 \mathrm{vs}$. pcDNA3.1 or si-NC. circRNA, circular RNA; DDP, cisplatin; $\mathrm{NC}$, negative control; OE, overexpression vector; si, small interfering RNA.

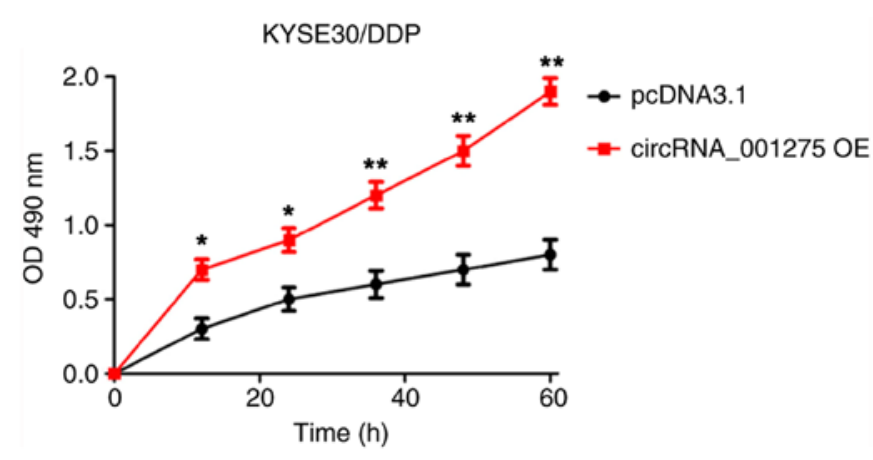

Figure 5. circRNA_001275 promotes cell proliferation. KYSE30/DDP, N=3. ${ }^{*} \mathrm{P}<0.05,{ }^{* *} \mathrm{P}<0.01$ vs. pcDNA3.1 or si-NC. circRNA, circular RNA; DDP, cisplatin; NC, negative control; OD, optical density; OE, overexpression vector; si, small interfering RNA.

miR-370-3p NC group (both $\mathrm{P}<0.05$; Fig. S1). A luciferase assay revealed that the relative luciferase activity in the circRNA_001275-wt and miR-370-3p mimic co-transfection group was significantly decreased compared with the circRNA_001275-mut or miR-370-3p NC groups (both $\mathrm{P}<0.05$; Fig. 7B). RT-qPCR revealed that miR-370-3p was downregulated in cisplatin-resistant cells compared with corresponding sensitive cells $(\mathrm{P}<0.05$; Fig. 7C); additionally, miR-370-3p levels were markedly decreased in cisplatin-resistant tissues compared with adjacent tissues. Furthermore, the expression of miR-370-3p was significantly decreased in the circRNA_001275 OE group, and significantly increased in the si-circRNA_001275 group, compared with the corresponding control groups (both $\mathrm{P}<0.05$; Fig. 7D). Therefore, the results demonstrated that circRNA_001275 may serve as a sponge for miR-370-3p.

Wnt7a is a direct target gene of miR-370-3p. The potential binding sites of miR-370-3p with Wnt7a-wt and Wnt7a-mut were predicted. The results indicated a potential binding site for miR-370-3p in the Wnt7a 3'-UTR (Fig. 8A). A luciferase assay revealed that the relative luciferase activity in the Wnt7a-3'-UTR-wt and miR-370-3p mimic co-transfection group was significantly decreased compared with the miR-370-3p NC or Wnt7a-3'-UTR-mut groups (both $\mathrm{P}<0.05$; Fig. 8B). RT-qPCR revealed that Wnt7a mRNA was significantly upregulated in cisplatin-resistant cells compared with corresponding sensitive cells (both $\mathrm{P}<0.05$; Fig. 8C); additionally, Wnt7a levels were markedly increased in cisplatin-resistant tissues compared with adjacent tissues. Furthermore, Wnt7a mRNA was significantly decreased in the miR-370-3p mimic group, and significantly increased in the miR-370-3p inhibitor group, compared with the miR-370-3p $\mathrm{NC}$ group (both $\mathrm{P}<0.05$; Fig. 8D). Additionally, the Wnt7a protein level was significantly decreased in the miR-370-3p mimic group, and significantly increased in the miR-370-3p inhibitor group, compared with the miR-370-3p NC group (both $\mathrm{P}<0.05$; Fig. 9A and B). Therefore, the results revealed that Wnt7a is a direct target gene of miR-370-3p.

Wnt7a is activated by the circRNA_001275/miR-370-3p axis. The effects of the circRNA_001275/miR-370-3p axis on Wnt7a expression were investigated. RT-qPCR revealed that Wnt7a mRNA expression was significantly increased in the circRNA_001275 OE group and significantly decreased in the si-circRNA_001275 group; however, these effects were attenuated by miR-370-3p mimic and inhibitor, respectively $(\mathrm{P}<0.05$; Fig. 10A). Furthermore, MTT results showed that overexpression of circRNA_141539 increased cell viability, and that circRNA_001275 silencing decreased cell viability; these effects were abrogated by miR-370-3p mimic and inhibitor, respectively ( $\mathrm{P}<0.05$; Fig. 10B).

\section{Discussion}

Previous studies have reported that circRNAs-miRNA-mRNA axes play important roles in tumorigenesis and development. For example, Xie et al (12) revealed that circ_001569 is upregulated in colorectal cancer. Similarly, Zhong et al (13) 
A
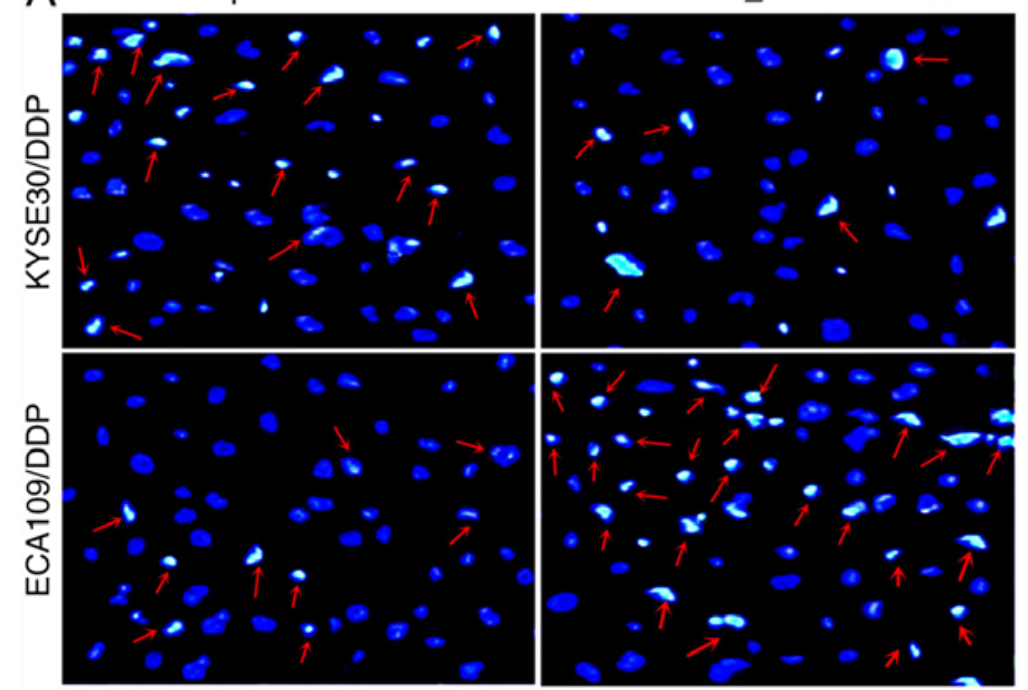

si-NC

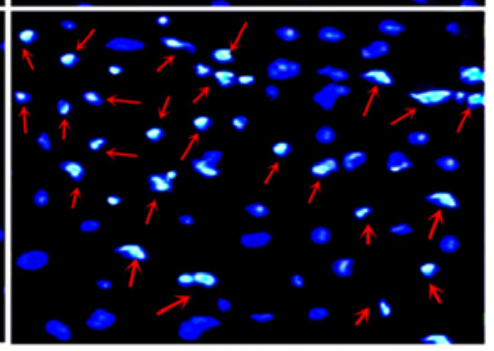

si-circRNA_001275
B

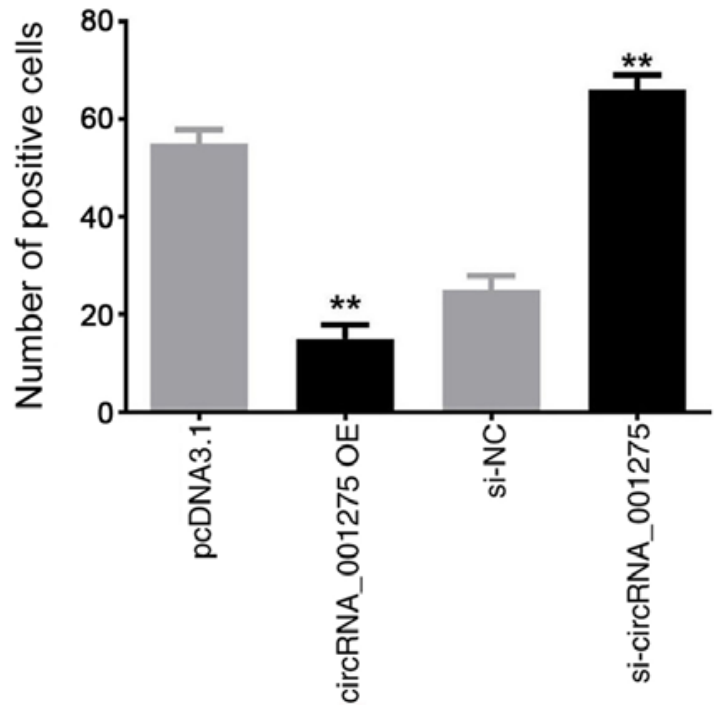

Figure 6. circRNA_001275 inhibits cell apoptosis (n=3). (A) Hoechst 33258 staining assay. (B) Number of positive cells. N=3. ${ }^{*} \mathrm{P}<0.05$, ${ }^{* *} \mathrm{P}<0.01$ vs. pcDNA3.1 or si-NC. circRNA, circular RNA; DDP, cisplatin; NC, negative control; OE, overexpression vector; si, small interfering RNA.
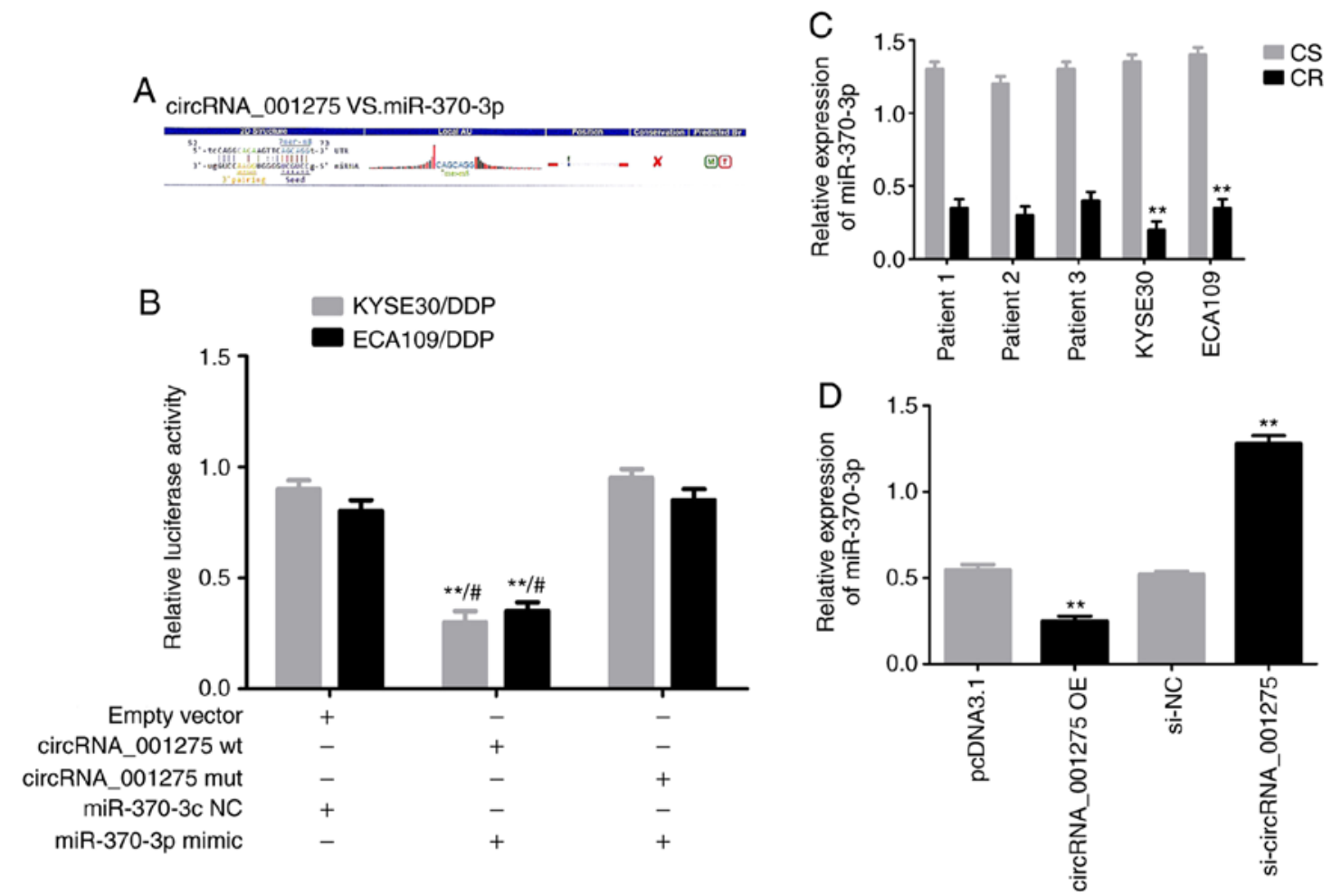

Figure 7. circRNA_001275 serves as an miR-370-3p sponge. (A) Schematic representation of potential binding sites of miR-370-3p with 3'-UTR of circRNA_001275. (B) Dual luciferase assays results. ${ }^{* *} \mathrm{P}<0.01$ vs. empty vector; ${ }^{*} \mathrm{P}<0.01$ vs. mut + mimic. (C) RT-qPCR analysis of miR-370-3p expression in tissues and cells. ${ }^{* *} \mathrm{P}<0.01$ vs. CS. (D) RT-qPCR analysis miR-370-3p expression in circRNA_001275 transfection groups. ${ }^{* *} \mathrm{P}<0.01$ vs. pcDNA3.1 or si-NC. circRNA, circular RNA; CR, cisplatin-resistant; CS, cisplatin-sensitive; DDP, cisplatin; miR/miRNA, microRNA; mut, mutated; NC, negative control; OE, overexpression vector; RT-qPCR, reverse transcription-quantitative PCR; si, small interfering RNA; UTR, untranslated region; wt, wild-type.

demonstrated that circRNA transcription factor 25 (circTCF25) is upregulated in bladder carcinoma, and that circTCF25 downregulates miR-103a-3p, increases cyclin-dependent kinase 6 expression and promotes proliferation. Li et al (14) found that circRNA RNA-binding motif, single-stranded-interacting protein 3 (circRBMS3) is upregulated in gastric cancer, and that increased circRBMS3 expression is associated with advanced tumor-node-metastasis stage. Gao et al (15) reported that the circ_0006528/miR-7-5p/Raf1 axis serves a key role in adriamycin-resistant breast cancer. Kun-Peng et al (16) 
A

Wnt7a vs.miR-370-3p

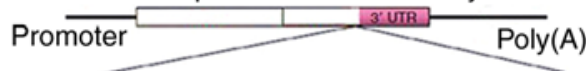

Wnt7a-3'-UTR-wt 5'-AGCGAACGCUUCUGCAGCAGGA-3' has-miR-370-3p 5'-UGGUCCAAGGUGGGGUCGUCCG-3' Wnt7a-3'-UTR-mut 5'-AGCGAACGCUUCUGGACCUGCA-3'

B

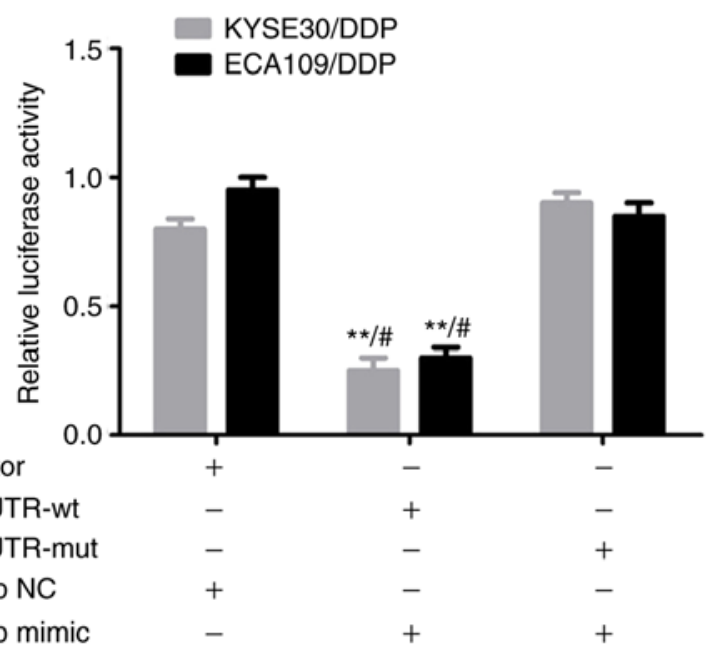

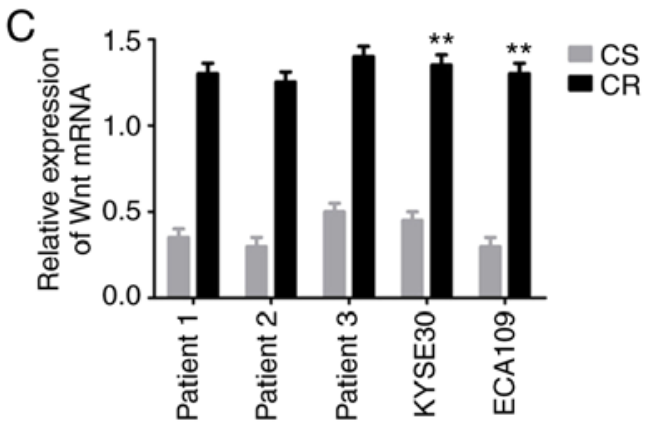

$\mathrm{D}$

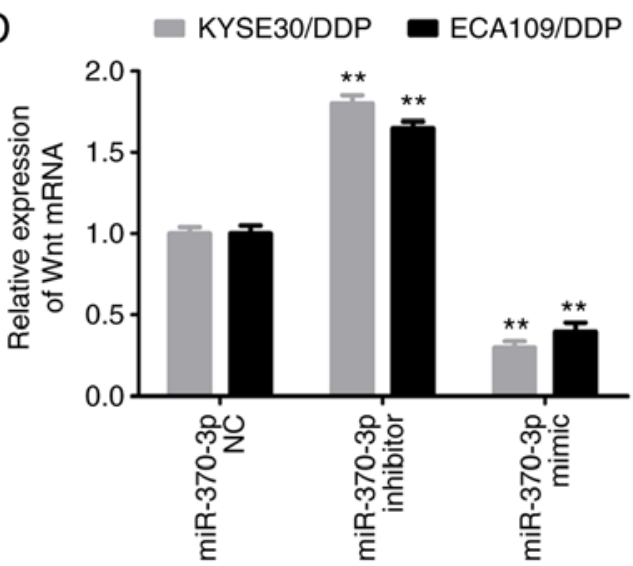

Figure 8. Wnt7a is a direct target of miR-370-3p. (A) Schematic representation showing that miR-370-3p specifically binds to the wt 3'-UTR sequence of Wnt7a. (B) Dual luciferase assays results. ${ }^{* *} \mathrm{P}<0.01$ vs. empty vector; ${ }^{*} \mathrm{P}<0.01$ vs. mut + mimic. (C) RT-qPCR analysis of Wnt7a mRNA expression in tissues and cells. ${ }^{* *} \mathrm{P}<0.01$ vs. CS. (D) RT-qPCR analysis of Wnt7a mRNA expression in circRNA_001275 transfection groups. ${ }^{* *} \mathrm{P}<0.01$ vs. pcDNA3.1 or si-NC. circRNA, circular RNA; CR, cisplatin-resistant; CS, cisplatin-sensitive; DDP, cisplatin; miR, microRNA; mut, mutated; NC, negative control; OE, overexpression vector; RT-qPCR, reverse transcription-quantitative PCR; siRNA, small interfering RNA; UTR, untranslated region; Wnt7a, Wnt family member 7A; wt, wild-type.

A

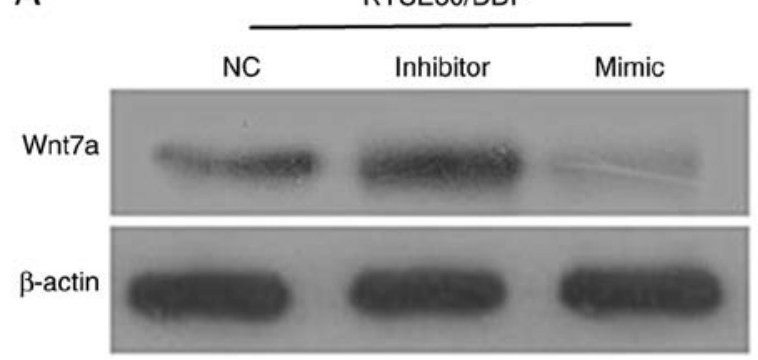

B

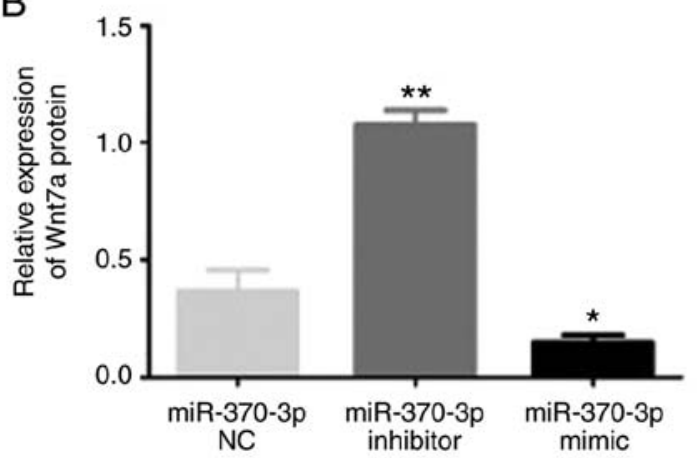

ECA109/DDP
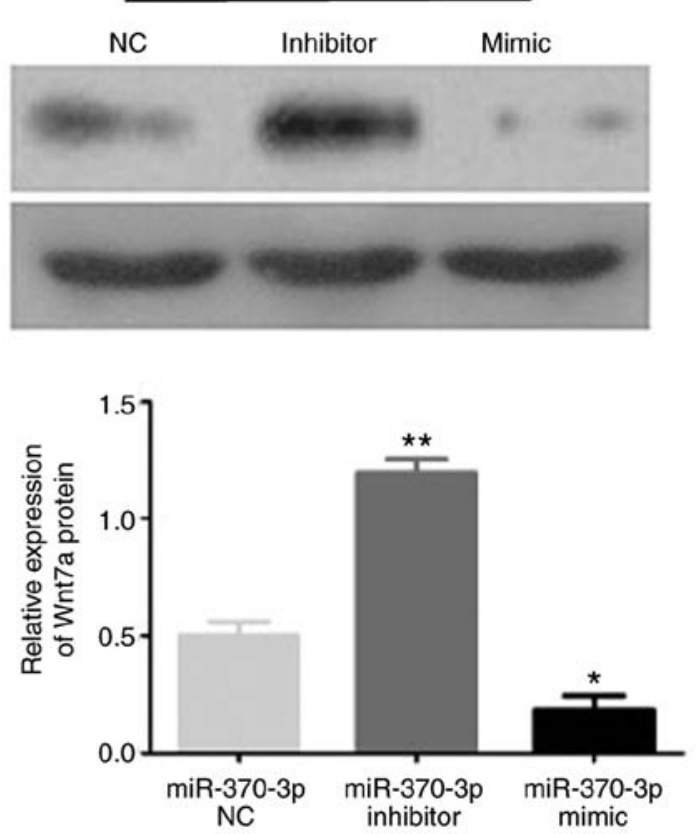

Figure 9. Wnt7a protein is regulated by miR-370-3p. (A) Western blot and (B) quantification of Wnt7a expression in DDP-resistant cells. ${ }^{*} \mathrm{P}<0.05$, ${ }^{* * *} \mathrm{P}<0.01$ vs. miR-370-3p. DDP, cisplatin; miR, microRNA; NC, negative control; Wnt7a, Wnt family member 7A.

reported that circRNA PVT1 promotes doxorubicin and cisplatin resistance in osteosarcoma by regulating ATP-binding cassette subfamily B member 1 expression. Dai et al (17) reported that circRNAs may be involved in epidermal growth 

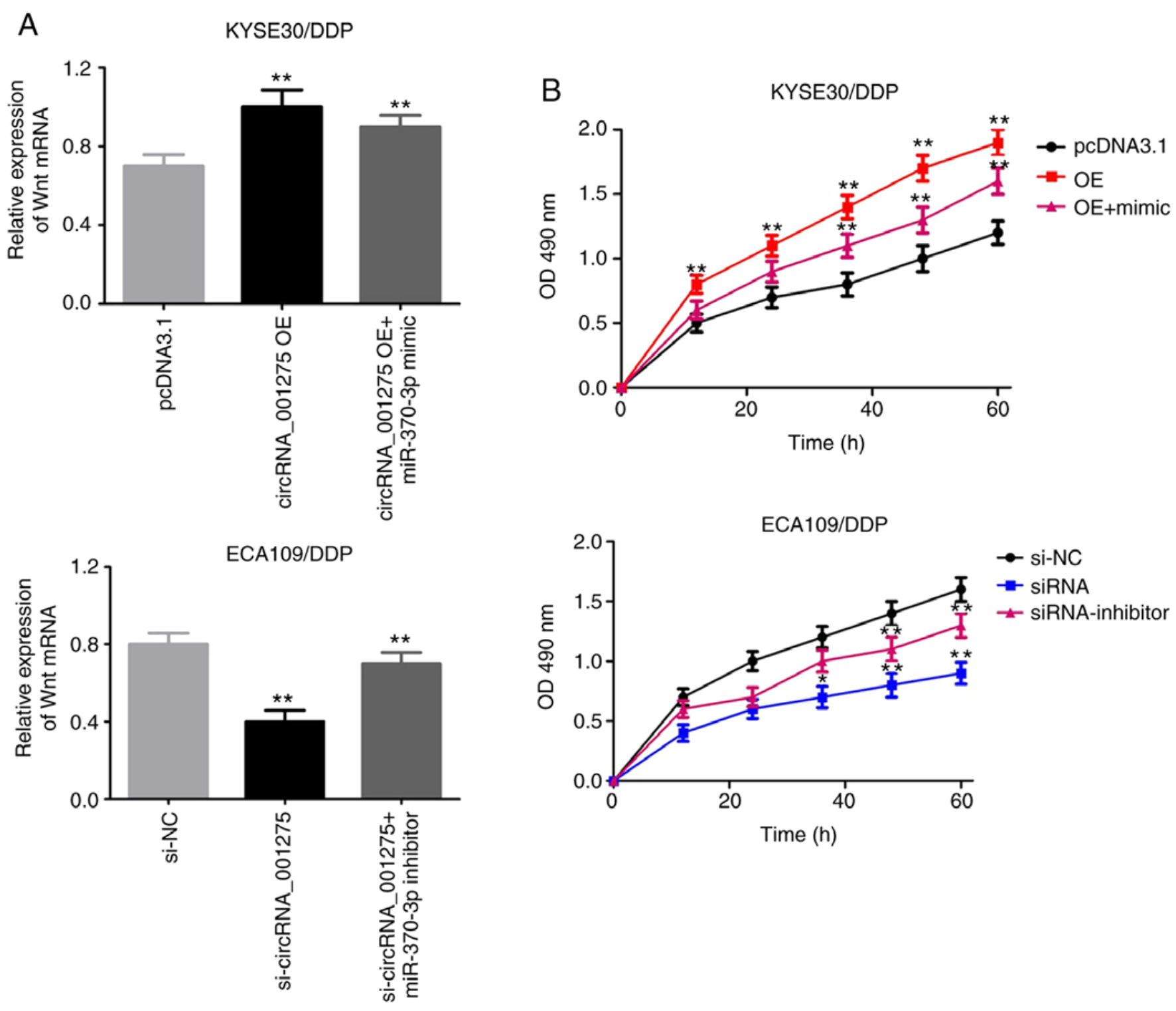

Figure 10. Wnt7a can activated by the circRNA_001275/miR-370-3p axis. (A) Reverse transcription-quantitative PCR analysis of Wnt7a expression in circRNA_001275 and miR-370-3p transfection groups. (B) MTT assay results. ${ }^{* *} \mathrm{P}<0.01$ vs. pcDNA3.1 or si-NC. circRNA, circular RNA; DDP, cisplatin; miR, microRNA; NC, negative control; OE, overexpression vector; siRNA, small interfering RNA. Wnt7a, Wnt family member 7; OD, optical density.

factor receptor-tyrosine kinase inhibitor resistance. However, the roles of circRNAs in cisplatin-resistant esophageal cancer remain unclear.

The present study revealed that circRNA_001275 was upregulated in cisplatin-resistant esophageal cancer tissues and cell lines. Furthermore, circRNA_001275 was found to promote the proliferation of cisplatin-resistant cells. To the best of the authors' knowledge, circRNA_001275 has only been investigated in a study by Zhao (18), who reported that circRNA_001275 was significantly upregulated in post-menopausal osteoporosis and may serve as a novel potential diagnostic biomarker. The present study revealed that circRNA_001275 may function as an oncogenic factor and serve as a promising biomarker for patients with cisplatin-resistant esophageal cancer.

The present study revealed that circRNA_001275 may act as an miR-370-3p sponge to upregulate Wnt7a expression. miR-370-3p is located at the $\Delta$-like homolog $1 / \mathrm{D} 103$ region of chromosome 14 . Several reports have shown that miR-370-3p may promote the progression of bladder cancer (19) and glioma (20), and affect temozolomide sensitivity in glioblastoma (21). Furthermore, the Wnt signaling pathway plays an important role in embryogenesis and cancer development by regulating the expression of genes involved in cell proliferation, differentiation and survival (22). Wnt7a, one of Wnt ligands, activates the canonical Wnt $/ \beta$-catenin signaling pathway to promote cell proliferation (23). Previous studies have revealed that $\mathrm{Wnt} 7 \mathrm{a}$ expression is dysregulated in several tumors, including gastric, lung, liver, pancreatic, colorectal, cervical and breast cancers (24,25). Wnt7a is a target gene of miR-150 (26), miR-34a (27) and miR-29b (28) in several types of cancer. The present study revealed that Wnt7a was significantly upregulated in cisplatin-resistant esophageal cancer. 
Therefore, miR-370-3p-induced Wnt7a upregulation may be one of the mechanisms underlying cisplatin resistance. However, whether the circRNA_001275/ miR-370-3p/Wnt7a axis serves as an independent or auxiliary factor driving cisplatin resistance, as well as the associated mechanisms, require further investigation.

To the best of our knowledge, the present study was the first to determine that circRNA_001275 was upregulated in cisplatin-resistant esophageal cancer, and that circRNA_001275 may promote cisplatin resistance by sponging miR-370-3p to upregulate Wnt7a expression. However, future studies investigating a larger number of samples are required to validate these results. Collectively, the results of the present study suggested that the circRNA_001275/miR-370-3p/Wnt7a axis may serve as a novel potential diagnostic biomarker and therapeutic target for patients with cisplatin-resistant esophageal cancer.

\section{Acknowledgements}

We thank Shanghai GeneChem Co., Ltd. for conducting and analyzing the circRNA chip assay and luciferase assay.

\section{Funding}

This work was supported by Chinese Postdoctoral Science Foundation (grant no. 2018M640266) and Postgraduate Independent Exploration and Innovation Project of Central South University (grant no. 2019zzts356).

\section{Availability of data and materials}

The datasets used and/or analyzed during the current study are available from the corresponding author on reasonable request.

\section{Author contributions}

ZHL, SX, and CHH designed the study and supervised the experiments. FWZ conducted the majority of the molecular and cellular experiments, data analysis, interpretation of data and preparation of the manuscript. SZY and WYL were responsible for bioinformatics and statistical analysis of the data. CYL and XHL participated in the interpretation of the data and preparation of the manuscript. All authors read and approved the final manuscript.

\section{Ethics approval and consent to participate}

The present study was approved by the Ethics Committee of The First Affiliated Hospital of China Medical University and conducted in accordance with the Declaration of Helsinki. Informed consent was obtained from all patients.

\section{Patient consent for publication}

Not applicable.

\section{Competing interests}

The authors declare that they have no competing interests.

\section{References}

1. Hall KA, Spicer MT, Ilich JZ and Levenson CW: Nutritional care for patients with esophageal cancer. Topics Clin Nutr 34: 2-13, 2019.

2. Su LL, Chang XJ, Zhou HD, Hou LB and Xue XY: Exosomes in esophageal cancer: A review on tumorigenesis, diagnosis and therapeutic potential. World J Clin Cases 7: 908-916, 2019.

3. Shi H, Mao Y, Ju Q, Wu Y, Bai W, Wang P, Zhang Y and Jiang M: $\mathrm{C}$-terminal binding protein-2 mediates cisplatin chemoresistance in esophageal cancer cells via the inhibition of apoptosis. Int $\mathbf{J}$ Oncol 53: 167-176, 2018.

4. Huang XP, Li X, Situ MY, Huang LY, Wang JY, He TC, Yan QH, Xie XY, Zhang YJ, Gao YH, et al: Entinostat reverses cisplatin resistance in esophageal squamous cell carcinoma via down-regulation of multidrug resistance gene 1. Cancer Lett 414: 294-300, 2018.

5. Sun Y, Zhai L, Ma S, Zhang C, Zhao L, Li N, Xu Y, Zhang T, Guo Z, Zhang H, et al: Down-regulation of RIP3 potentiates cisplatin chemoresistance by triggering HSP90-ERK pathway mediated DNA repair in esophageal squamous cell carcinoma. Cancer Lett 418: 97-108, 2018.

6. Meng X, Li X, Zhang P, Wang J, Zhou Y and Chen M: Circular RNA: An emerging key player in RNA world. Brief Bioinform 18: 547-557, 2017.

7. Chen LL and Yang L: Regulation of circRNA biogenesis. RNA Biol 12: 381-388, 2015.

8. Huang $\mathrm{C}$ and Shan $\mathrm{G}$ : What happens at or after transcription: Insights into circRNA biogenesis and function. Transcription 6: 61-64, 2015.

9. Panda AC: Circular RNAs Act as miRNA sponges. Adv Exp Med Biol 1087: 67-79, 2018.

10. World Medical Association Inc: Declaration of Helsinki. Ethical principles for medical research involving human subjects. J Indian Med Assoc 107: 403-405, 2009.

11. Livak KJ and Schmittgen TD: Analysis of relative gene expression data using real-time quantitative PCR and the 2(-Delta Delta C(T)) method. Methods 25: 402-408, 2001.

12. Xie H, Ren X, Xin S, Lan X, Lu G, Lin Y, Yang S, Zeng Z, Liao W, Ding YQ and Liang L: Emerging roles of circRNA_001569 targeting miR-145 in the proliferation and invasion of colorectal cancer. Oncotarget 7: 26680-26691, 2016.

13. Zhong Z, Lv M and Chen J: Screening differential circular RNA expression profiles reveals the regulatory role of circTCF25-miR-103a-3p/miR-107-CDK6 pathway in bladder carcinoma. Sci Rep 6: 30919, 2016.

14. Li G, Xue M, Yang F, Jin Y,Fan Y and Li W: CircRBMS3 promotes gastric cancer tumorigenesis by regulating miR-153-SNAI1 axis. J Cell Physiol 234: 3020-3028, 2019.

15. Gao D, Zhang X, Liu B, Meng D, Fang K, Guo Z and Li L: Screening circular RNA related to chemotherapeutic resistance in breast cancer. Epigenomics 9: 1175-1188, 2017.

16. Kun-Peng Z, Xiao-Long $M$ and Chun-Lin Z: Overexpressed circPVT1, a potential new circular RNA biomarker, contributes to doxorubicin and cisplatin resistance of osteosarcoma cells by regulating ABCB1. Int J Biol Sci 14: 321-330, 2018.

17. Dai Y, Huang Y, Huang J, Wen C, Zhou H and Wu L: Differential expression of circular RNAs in gefitinib-acquired resistant non-small cell lung cancer cells. Tumor 37: 1128-1135, 2017.

18. Zhao K: Study on the expression profile of cyclic RNA in postmenopausal osteoporosis and screening of potential molecular markers (unpublished $\mathrm{PhD}$ thesis). Southern Medical University, 2018.

19. Huang X, Zhu H, Gao Z, Li J, Zhuang J, Dong Y, Shen B, Li M, Zhou $\mathrm{H}$, Guo $\mathrm{H}$, et al: Wnt7a activates canonical Wnt signaling, promotes bladder cancer cell invasion, and is suppressed by miR-370-3p. J Biol Chem 293: 6693-6706, 2018.

20. Peng Z, Wu T, Li Y, Xu Z, Zhang S, Liu B, Chen Q and Tian D: MicroRNA-370-3p inhibits human glioma cell proliferation and induces cell cycle arrest by directly targeting $\beta$-catenin. Brain Res 1644: 53-61, 2016.

21. Gao YT, Chen XB and Liu HL: Up-regulation of miR-370-3p restores glioblastoma multiforme sensitivity to temozolomide by influencing MGMT expression. Sci Rep 6: 32972, 2016.

22. Anastas JN and Moon RT: WNT signalling pathways as therapeutic targets in cancer. Nat Rev Cancer 13: 11-26, 2013.

23. Tríbulo P, Jumatayeva G, Lehloenya K, Moss JI, Negrón-Pérez VM and Hansen PJ: Effects of sex on response of the bovine preimplantation embryo to insulin-like growth factor 1, activin A, and WNT7A. BMC Dev Biol 18: 16, 2018. 
24. Wang W, Yan X, Lin Y, Ge H and Tan Q: Wnt7a promotes wound healing by regulation of angiogenesis and inflammation: Issues on diabetes and obesity. J Dermatol Sci, Feb 15, 2018 (Epub ahead of print)

25. Liu Y, Dong Y, Zhao L, Su L and Luo J: Circular RNA-MTO1 suppresses breast cancer cell viability and reverses monastrol resistance through regulating the TRAF4/Eg5 axis. Int $\mathrm{J}$ Oncol 53: 1752-1762, 2018.

26. MacLean JA II, King ML, Okuda H and Hayashi K: WNT7A regulation by miR-15b in ovarian cancer. PLoS One 11: e0156109, 2016

27. Feng G, Fan Y, Li L, et al: MicroRNA-34a sensitizes breast cancer cells to paclitaxel treatment by targeting WNT7A. Bachu Med J 15: 6-10, 2018 (In Chinese).
28. Avasarala S, Van Scoyk M, Wang J, Sechler M, Vandervest K, Brzezinski C, Weekes C, Edwards MG, Arcaroli J, Davis RE, et al: hsa-miR29b, a critical downstream target of non-canonical Wnt signaling, plays an anti-proliferative role in non-small cell lung cancer cells via targeting MDM2 expression. Biol Open 2: 675-685, 2013.

(i) (3) This work is licensed under a Creative Commons Attribution-NonCommercial-NoDerivatives 4.0 International (CC BY-NC-ND 4.0) License. 